\title{
Structure-based drug design and AutoDock study of potential protein tyrosine kinase inhibitors
}

\author{
Hamed Ismail Ali ${ }^{1}$, Tomofumi Nagamatsu ${ }^{2}$, Eiichi Akaho ${ }^{3 *}$
}

${ }^{1}$ Department of Pharmaceutical Chemistry, Helwan university, Helwan, Egypt; ${ }^{2}$ Division of Pharmaceutical Sciences, Graduate School of Medicine, Dentistry and Pharmaceutical Sciences, Okayama University, 1-1-1 Tsushima-Naka, Okayama 700-8530, Japan; ${ }^{3}$ Faculty of Pharmaceutical Sciences, Kobe Gakuin University, 1-1-3, Minatojima, Chuo-ku, Kobe 650-8586, Japan; Eiichi Akaho - Phone: +81 78974 4845; Fax: +81 78 974 4845; E-mail: akaho@pharm.kobegakuin.ac.jp; *Corresponding author.

Received January 04, 2011; Accepted January 06, 2011; Published February 07, 2011

\section{Abstract:}

Different classes of compounds were investigated for their binding affinities into different protein tyrosine kinases (PTKs) employing a novel flexible ligand docking approach by using AutoDock 3.05 and 4. These compounds include many flavin analogs, which were developed in our group with varying degrees of cytotoxic activity (comparable or moderately superior to cisplatin and ara-c), and database selected analogs. They were docked onto twelve different families of PTKs retrieved from the Protein Data Bank. These proteins are representatives of plausible models of interactions with chemotherapeutic agents. A comparative study of the intact co-crystallized ligands of various types of PTKs was carried out. Results revealed that the new class of 5-deazapteridine and steroid hybrid compounds VIa,b, and d, and the vertical-type bispyridodipyrimidine with n-hexyl chain junction between its $\mathrm{N}-10$ and $\mathrm{N}-10$ atoms Xa, exhibited non-selective PTK binding capacities, with the lowest Gb. On the other hand, 2-amino benzoic acid analog IIa, phenoxypyrido [3, 4-d]pyrimidine derivative IVc, tyrosine containing tripeptide Vd, and the one from Sumisho data base 831 are proposed to have selective PTK binding affinities to certain classes of tyrosine kinases, namely, HGFR (c-met), ZAP-70, insulin receptor kinase, EGFR, respectively. All These compounds of highest affinities were docked within the binding sites of PTKs with reasonable RMSD and 1-5 hydrogen bonds.

\section{Background:}

In 2009, two kinase inhibitors were approved for the treatment of cancer; and another for the treatment of renal cell carcinoma [1]. Protein-tyrosine kinases (PTKs) play an important role in the control of most fundamental cellular processes including the cell cycle, cell migration, cell metabolism, and cell survival, as well as cell proliferation and differentiation [2]. Docking of the molecules into their respective 3D macromolecular targets is a widely used method for lead optimization [3]. Docking programs find their most important applications in virtual database screening approaches in which hundreds of thousands of molecules are docked into the binding pocket to identify plausible binders [4]. It was reported that docking programs are able to predict experimental poses with deviation average from 1.5 to $2.0 \AA$ root mean square deviation (RMSD) [5]. One of the most well-known docking programs is Autodock [6]. Structure-based drug design methods utilize knowledge of the three-dimensional structure of a receptor complexed with a lead molecule in an attempt to optimize the bound ligand or a series of congeneric molecules. Using a model with a given structure, a medicinal chemist can compute an activity of a molecule [7].

Development of PTK signaling inhibitors has evolved into an important approach toward new therapeutics [8]. Tyrosine kinase inhibitors as a target for anti-angiogenesis can be aptly applied as a new mode of cancer therapy [9]. The role of tyrosine kinases in cancer molecular pathogenesis is immense and recently tyrosine kinases have come in vogue as potential anticancer drug targets, sending a number of anticancer drugs to the market. Several tyrosine kinase inhibitors are undergoing human trials and several are in the pipeline of drug discovery [10].
The main objective of this study is to design and to propose potential tyrosine kinase inhibitors from synthesized compounds and databaseselected analogs. In this study twelve different target protein tyrosine kinases were selected to investigate the potential binding affinities within their corresponding binding sites. Those include fibroblast growth factor receptor, epidermal growth factor receptor, hepatocyte growth factor receptor, insulin receptor kinase, sarcoma proto-oncogenic kinase, spleen tyrosine kinase, platelet-derived growth factor receptor, vascular endothelial growth factor receptor, Abelson tyrosine kinase, hemopoietic cell kinase, Zeta-chain-associated protein kinase, and Janus kinase.

The selective PKC inhibitory activities of 5-deazaflavins, 2-deoxy-2phenyl-5-deazaflavins, \{2-phenylpyrimido[4, 5-b]quinolin-4(10H)-ones \}, and their effective growth inhibition against cancer cells such as the A431 cells, and HT1080 cells were reported [11]. Synthesis of the hybrid compounds, which structurally contain two different biologically or pharmacologically active compounds of 5-deazaflavins and steroids in the same ring system, were carried out by condensation of 6-(monosubstituted amino)uracils or 6-(monosubstituted amino)-2-phenylpyrimidin-4(3H)ones with 2-hydroxy-methylene androstanolone or 2hydroxymethylenetestosterone. Eventually, their potential anti-coccidiosis activities were reported [12]. These compounds were synthesized with the rationale that steroids can penetrate the cell membrane, and bind the cell nucleus. Steroid receptors may serve to localize and concentrate appended drug species, mainly in hormones responsive for the production of neoplastic cells. Moreover, 5-deazaflavins (5-deazaisoalloxazines) have greatly attracted our attention [13-17]. Antitumor activities of nitro-5deazaflavin-pyrrolecarboxamide hybrid molecules [18] and 5-amino-5- 
deazaflavin derivatives revealed potential inhibitory activities against L1210 or KB cells [19].

\begin{abstract}
Methodology:
AutoDock protocol:

Firstly, all bound waters, ligands and cofactors were removed from the proteins. The macromolecule was checked for polar hydrogens, partial atomic Kollman charges were assigned, and then atomic solvation parameters were allotted. Torsion bonds of the inhibitors were selected and defined. Secondly, the three dimensional grid box was created by AutoGrid algorithm to evaluate the binding energies on the macromolecule coordinates. The grid maps representing the intact ligand in the actua docking target site were calculated with AutoGrid (part of the AutoDock package). The three dimensional grid box with $60 \AA$ grid size (x, y, z) with a spacing of $0.300 \AA$, grid was created. Eventually cubic grids encompassed the binding site where the intact ligand was embedded. Finally, AutoDock was used to calculate the binding free energy of a given inhibitor conformation in the macromolecular structure while the probable structure inaccuracies were ignored in the calculations.
\end{abstract}

\section{Target macromolecules investigated:}

Twelve different target protein tyrosine kinases were investigated, and those were retrieved from the Protein Data Bank, http://www.rcsb.org/pdb/home/home.do. For each docking target, crucial amino acids of the active site were identified using data in PDBsum, http://www.ebi.ac.uk/pdbsum/.

\section{Compounds involved in this study:}

The compounds investigated in this study include 2, 3, 4-trisubstitutred1, 2-dihydropyrrolo[1, 2-a][1, 3, 5]triazin derivatives Ia-k, 4-(3, 5-di-tertbutylphenylcarbamoyl)-2-amino benzoic acid, or 2-nitro benzoic acid, or their methylester analogs IIa-d, benzopyrido, benzopyrimido, or benzopiprazino[1, 4]dioxin-2-ol IIIa-c and dipyrido[1, 4] dioxin-2-ol analogs IIId-g, 2-substituted-(6-fluoro-3, 4-dihydro-4-substituted phenoxypyrido[3, 4-d]pyrimidin-2-yl) derivatives IVa-d, tyrosine containing dior tripeptides Va-f, acetylated tyrosine containing tetrapeptides analogs Vg-m, deazapteridine-steroid hybrid compounds VIa-d, as a new class of the hybrid compounds possessing 5-deazapteridine and steroid in the same ring system, synthesized by condensation of 6 -(monosubstituted amino) uracils or 6-(monosubstituted amino)-2-phenylpyrimidin-4(3H)-ones with 2-hydroxy-methylene androstanolone or 2-hydroxymethyl- enetestosterone under heating in the presence of p-toluenesulfonic acid monohydrate.12 5deazaflavin derivatives including: 2-deoxo-2-phenyl-5-deazaflavins VIIah,13,142-deoxo-2-piperidino-5-deazaflavin VIIi,15 2-deoxo-2-morpholino5-deazaflavin VIIj,15 new 2-deoxo-2-phenylflavins VIIm-o, computationally newly designed 2-deoxo-2-glycino, 2-tyrosino, and 2histidi- no-N10-methylflavin-N-oxides VIIp-r, respectively, new 5deazaflavin derivatives VIIIa-c, N3-methyl -5-(monosubstituted alkylamino)-5-deazaflavin derivatives VIIId-g, and 14 computationally designed pyridodipyrimidines IXa-f, 14 bis-(5-deazaflavin-10-yl) alkanes $\mathrm{Xa}, 14$ and Xb. Additional compounds were obtained from Sumisho data base (Figure 1). Those include abacavir, PB-01547328, PB-99211665, F0007-0958, OSSK-633719, OSSK-692604, 831, PB-00623451, and compound 1505335.

Analysis of the docking results:

Accelrys DS modeling 1.7 software [DS modeling 1.7; Accelrys Inc., San Diego, CA, (2006), www.accelrys.com], and Accelrys DS Visualizer 2.5 were utilized for molecular modeling, for evaluation of hydrogen bonds in ligand-receptor interaction, and for measuring RMSD which was computed and expressed in angstrom $(\AA)$ as a locational comparison of two molecules of interest. In our particular study RMSD was measured as distance between the centroids of the docked inhibitor and the intact ligand.

\section{Results and Discussion:}

We investigated the AutoDock binding affinities of various structures for their potential protein tyrosine inhibitory activities, including: 2, 3, 4- trisubstitutred -1, 2-dihydropyrrolo[1, 2-a][1, 3, 5]triazin derivatives Ia-k, 4-(3, 5-di-tert-butylphenylcarbamoyl)-2-amino benzoic acid, its 2-nitro benzoic acid, their methylester analogs IIa-d such as benzopyrido, benzopyrimido, benzopiprazino [1,4] dioxin-2-ol IIIa-C, dipyrido[1, 4] dioxin-2-ol analogs IIId-g, 2-substituted-(6-fluoro-3,4-dihydro-4substituted pheno-xypyrido [3, 4-d] pyrimidin-2-yl) derivatives IVa-d, tyrosine containing di- or tripeptides Va-f, acetylated tyrosine containing tetrapeptides analogs Vg-m, deazapteridine-steroid hybrid compounds VIa$\mathrm{d}$, hybrid compounds possessing 5-deazapteridine and steroid in the same ring system [12], 2-deoxo-2-phenyl-5-deazaflavins VIIa-h [13, 14], 2 deoxo-2-piperidino-5-deazaflavin VIIi [15], 2-deoxo-2-morpholino-5deazaflavin VIIj [15], 2-deoxo-2-phenylflavins VIIm-o, computationally newly designed 2-deoxo-2-glycino, 2-tyrosino, 2-histidino-N10methylflavin-N-oxides VIIp-r, 5-deazaflavin derivatives VIIIa-c, N3methyl-5-(monosubstituted alkylamino)-5-deazaflavin derivatives VIIId-g [14], computationally designed pyridodipyrimidines IXa-f [14], and bis(5-deazaflavin-10-yl) alkanes Xa,b [14]. Additional structures obtained from Sumisho Data Base were studied, including abacavir, PB-01547328, PB-99211665, F0007-0958, OSSK-633719, OSSK-692604, 831, PB00623451, and compound 1505335.

Molecular docking study:

Computer simulated automated docking studies were performed using the widely distributed molecular docking software, AutoDock versions 3.05 and 4.0, grid-based docking programs [6], which was utilized for the study of binding mode of the suggested potential inhibitors within different tyrosine kinases. In AutoDock, the overall docking energy of a given ligand molecule is expressed as the sum of intermolecular interaction energies including van der Waals attractive and repulsive energies, $\mathrm{H}$ bond interaction energy, coulombic electrostatic energy, and the internal steric energy of the ligand [4]:

\section{Evaluation of docking performance and accuracy:}

As shown in Table 1 (see Supplementary material), the RMSD values for the representative examples range from $0.07-1 \AA$ to $16 \AA$. It was found that the docked ligands are exactly superimposed with the originally embedded intact ligands not only for the best-scored conformations, but also for all the docked conformations. The docked compound (PRC1), 1terbutyl-3-p-tolyl-1h-pyrazolo[3, 4-d]pyrimidin-4-ylamine ligand, exhibited the best RMSD of $0.07 \AA$, and a low binding free energy ( $\mathrm{Gb}$ ) of $-11.56 \mathrm{Kcal} / \mathrm{mol}$. The representative examples of PTKs exhibited fairly reasonable docking results well comparable to their originally embedded intact ligands. Hence the obtained docking results were well correlated with the biological methods [20]

AutoDock binding affinities of the synthesized and designed compounds into PTKs -overview:

The molecular docking study revealed that many of the docked compounds currently studied exhibited the fairly good docking results into different PTKs in comparison with their originally embedded intact co-crystalized ligands. These promising compounds as drugs include: 4-(3, 5-di-tertbutylphenylcarbamoyl)-2-amino benzoic acid IIa, 4-(3, 5-di-tertbutylphenylcarbamoyl)-2-nitro benzoic acid IIb, 2-(1-piprazin-ylethyl)-6fluoro-3, 4-dihydro-4-(3, 4-dihydroxy) phenoxypyrido [3, 4-d]pyrimidine IVc, tyrosine containing tripeptide; Trp-Glu-Tyr-OH Vd, 3'and/or8'disubstituted-5'-deaza-androsta[2, 3-g]pteridine-2'， 4'(3'H,8'H)-diones VIa,b, and d, 5-deazaflavin derivatives including: 2-deoxo-2-phenyl-10methyl-5-deazaflavin-7-carboxylic acid VIIa, 2-deoxo-2-piperidino-7,10dimethyl-5-deazaflavin VIIi, 2-deoxo-2-tyrosino-5-deazaflavin VIIl, designed 2-deoxo-2-tyrosino, and 2-histidino-N10-methylflavin-N-oxides VIIq,r, N3-phenyl-8-chloro-N10-(3,4-xylyl)-5-deazaflavin VIIIb, N10dodecyl-5-deazaflavin VIIIc, N3-methyl-5-phenethyl-N10-n-octyl or ndodecyl-5-deazaflavin derivatives VIIIf,g, designed 2, 3, 8, 10-tetraphenylpyridodipyrimidines IXc, vertical bis-(5-deazaflavin-10-yl)-n-hexane Xa, and structure database coded 831 . 


\section{Bioinformation Volume 5}

open access

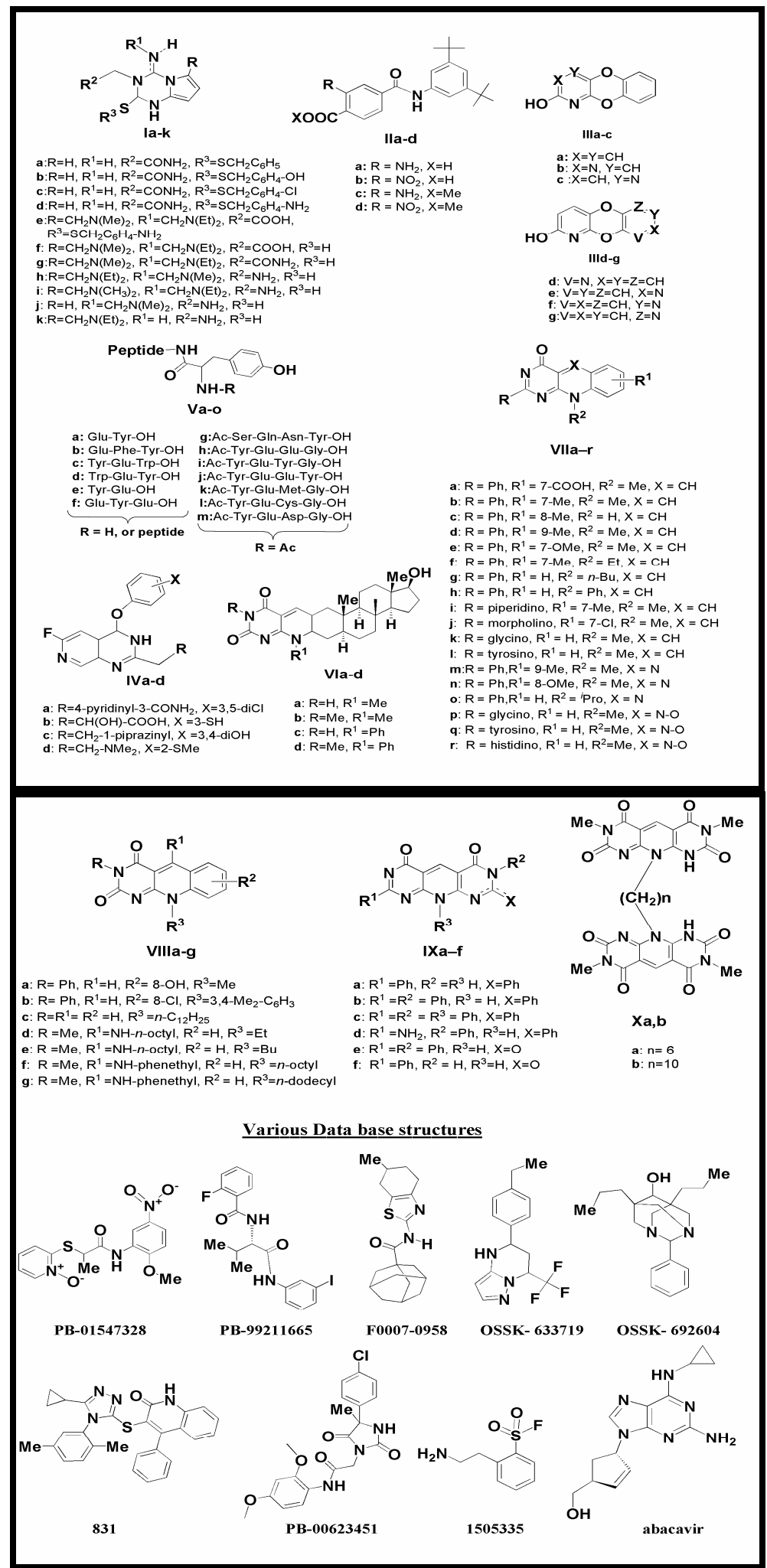




\section{Bioinformation Volume 5}

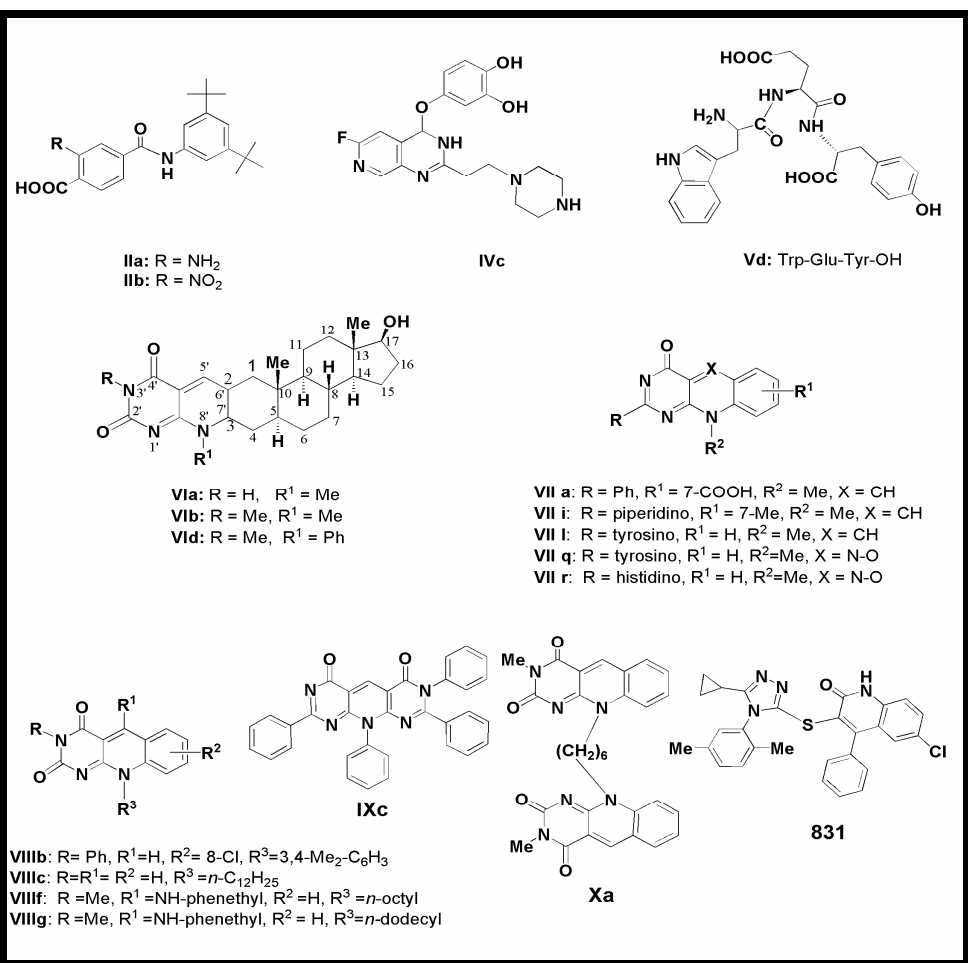

Figure 1: Structures of the compounds tested for the tyrosine kinase inhibition by computer docking

Docking evaluation for FGFR inhibitors:

The designed compound VIIq, 2-deoxo-2-tyrosino-N10-methylflavin-Noxides, was docked against FGFR (pdb code: 1agw) occupying the site in which the adenine of adenosine triphosphate binds, and the binding free energy ( $\mathrm{Gb}$ ) was $-11.85 \mathrm{kcal} / \mathrm{mol}$, with RMSD of $5.44 \AA$, where it was slightly deviated from the originally embedded intact ligand, indolinone (SU4984).

\section{Docking evaluation for EGFRK inhibitors:}

Compound IVc, 2-(1-piprazinylethyl)-6-fluoro-3, 4-dihydro-4-(3, 4dihydroxy) phenoxy pyrido[3, 4-d]pyrimidine, was bound into EGFRK (pdb code: 1m17) and exactly superimposed on erlotinib (TarcevaTM) currently marketed for the treatment of non-small cell lung cancer. The binding free energy ( $\mathrm{Gb}$ ) was reasonably as small as $-10.77 \mathrm{kcal} / \mathrm{mol}$, and five hydrogen bonds were formed via its polar 3, 4-dihydroxy and 2pirazinyl N-H groups.

\section{Docking evaluation for SRC inhibitors:}

The nonreceptor tyrosine kinase SRC (Pp60v-src; pdb code: 1skj) which, is considered to be causative protein for breast cancer and osteoporosis, was investigated by docking of the hybrid steroidal molecule, 8'-methyl-5'deaza-androsta [2, 3-g]pteridine-2', 4'(3'H,8'H)-diones VIa. The docked compound VIa penetrated to the intracellular Src SH2 domain target, exerting four hydrogen bonds between the pair of its 2-oxo, $\mathrm{N3H}$, and $17 \mathrm{OH}$, and amino acids of binding site, namely Arg62, Ile71, and Arg32, respectively as shown in Table 1(see Supplementary material).

Docking evaluation for Syk inhibitors:

The docked compound VIIb, 3', 8'-dimethyl-5'-deaza-androsta[2, 3g]pteridine-2', 4'(3'H, 8'H)-diones, was bound deeply into non-receptor spleen tyrosine kinase (SYK; pdb code:1xbb) into the activation loop typically to the intact ligand, Gleevec (STI-1, Imatinib) within 2.24 RMSD. The binding free energy was $-11.58 \mathrm{kcal} / \mathrm{mol}$, involving three hydrogen bonds via its polar moieties, 2-oxo and 17-OH groups.

ISSN 0973-2063 (online) 0973-8894 (print)

Bioinformation 5(9): 368-374 (2011)
Docking evaluation for HGFR inhibitors:

2-deoxo-2-phenyl-10-methyl-5-deazaflavin-7-carboxylic acid VIIa was shown to be potentially bound deeply within hepatocyte growth factor receptor (HGFR/c-Met; pdb code: 1r0p); with low binding free energy $(\Delta \mathrm{Gb})$ being $-11.13 \mathrm{kcal} / \mathrm{mol}$, and with low inhibition constant (Ki) being 6.98E-09. It exhibited two hydrogen bonds between the pair of its 7$\mathrm{COOH}, 7-\mathrm{C}=\mathrm{O}$ and $\mathrm{O}$ of Asp1231 and $\mathrm{HN}$ of Lys1232, with their distances being $1.88 \AA$, and $1.99 \AA$, respectively, while its RMSD was $3.11 \AA$ from the intact ligand, K-252a. Vertical bis-(N3-methyl-5deazaflavin-10-yl)-n-hexane Xa, was unselectively bound into HGFR/cMet (pdb code: $1 \mathrm{r} 0 \mathrm{p}$ ), which is considered to be an important cancer drug target, as there is a strong link between aberrant c-Met activity and oncogenesis [20].

\section{Docking evaluation for ABL inhibitors:}

The activation of ABL tyrosine kinanse causes chronic myelogenous leukemia (CML). Therefore, inhibitors of this PTK is proposed to be effective in the treatment of CML. Thus herein compound VIIIb, N3phenyl-8-chloro-N10-(3, 4-xylyl)-5-deazaflavin, was tested for docking into Abelson tyrosine kinase (ABL, pdb code: $1 \mathrm{fpu}$ ) to find potential inhibitor candidate. Compound VIIIb exerted binding free energy ( $\mathrm{Gb}$ ) of $-11.09 \mathrm{kcal} / \mathrm{mol}$, with RMSD of $4.49 \AA$, and one hydrogen bond between its 2-oxo group and $\mathrm{OH}$ of Thr315 was formed.

\section{Docking evaluation for PDFGR inhibitors:}

The activity of the c-Kit receptor protein-tyrosine kinase is tightly regulated in normal cells, whereas the deregulated c-Kit kinase activity is implicated in the pathogenesis of human cancers Based on its finding, compound VIIl, 2-deoxo-2-tyrosino-5-deazaflavin, was studied for its potential binding into platelet-derived growth factor receptor (PDGFR,ckit, pdb code: 1t46) as shown in Table 1. It was found that the docked compound VIIl was bound to Tyr670 identically to the intact ligand STI571 by two hydrogen bonds via 2-tyrosinyl $\mathrm{COOH}$ group, exerting binding free energy $(\Delta \mathrm{Gb})$ of within $-11.32 \mathrm{kcal} / \mathrm{mol}$. 
Docking evaluation for VEGFR inhibitors:

Compound VIIIf, N3-methyl-5-phenethyl-N10-n-octyl-5-deazaflavin, was docked into vascular endothelial growth factor receptor (VEGFR-2; pdb code: 1y6a) and showed two hydrogen bonds via its 4-oxo group with HN of Asn 921. It exhibited the best RMSD among this series of compounds.

Docking evaluation for ZAP-70 inhibitors:

The ZAP-70 tyrosine kinase plays a critical role in T cell activation as well as in the immune response, and thus it has become a logical target for immunomodulatory therapies. Based on this background, against ZAP-70 tyrosine kinase (pdb code: 1u59) compound IIb, 4-(3, 5-di-tertbutylphenylcarbamoyl)-2-nitro benzoic acid, was docked within the binding site resulting in a strong binding affinity.

\section{Docking evaluation for insulin receptor kinase inhibitors:}

Tyrosine containing tripeptide, Trp-Glu-Tyr-OH Vd is structurally similar to the structure of the intact ligand of the insulin receptor kinase (pdb code: 1rqq), thiophosphoric acid o-(adenosyl-phospho)phospho)-sacetamidyl-diester (112 E), where there is a ring equivalence between the indole and adenine rings of both compounds. In addition, the structura similarity exists between of tripeptides of $\mathrm{Vd}$ and triphosphate moiety of 112E. The docked compound Vd was exactly superimposed on and identically to the intact ligand within $1.32 \AA$ RMSD exhibiting five hydrogen bonds between the pair of its $\mathrm{COOH}$ groups, indolyl- $\mathrm{NH}$, and phenoxy-p-OH and amino acids of the binding sites, Gln1004, Lys1030, Met1079, and Ser1006, respectively, as shown in Table 1(see Supplementary material).

\section{Docking evaluation for HCK inhibitors:}

The docked compound VIIIg, was shown to be superimposed with the intact ligand PP1-532 of non-receptor tyrosine kinase, (HCK; pdb code: $1 \mathrm{qcf}$ ) and was bound at the ATP-binding site of the kinase, exhibiting one hydrogen bond with lys295. Its lipophilic n-dodecyl moiety comparable to the p-tolyl moiety of intact ligand PP1-532 was inserted into an adjacent hydrophobic pocket created by VaL281, Lys295, Ile336, and Asp404.

\section{Docking evaluation for JAK3 inhibitors:}

Compound VId, 5-deazapteridine and steroid hybrid compound, namely, 3'-methyl, 8'-phenyl-5'-deaza-androsta[2, 3-g]pteridine-2', 4'(3'H, 8'H)diones, was bound firmly against non-receptor tyrosine kinase, Janus kinase (JAK3; pdb code: 1yvj). Janus kinase is a central mediator of cytokine signaling. This provides a detailed view of the Jak3 active site, where VId exhibits one hydrogen bond between its 17-OH group and $\mathrm{O}$ of gly831 within RMSD of $2.10 \AA$.

\section{Docking evaluation for tested PTK inhibitors in general:}

To summarize all the aforementioned docking results, it was found that 19 compounds out of 85 exhibited noteworthy docking results. Those compounds include 4-substituted-2-amino and 2-nitro benzoic acid analogs IIa,b, 2, 4, 6-substituted phenoxypyrido[3, 4-d]pyrimidine IVc, tyrosine containing tripeptide Vd, 5-deazaandrosta [2, 3-g]pteridine derivatives VIa, b, d, 5-deazaflavin and flavin-N-oxide derivatives VIIa, i, l, q, r, N3phenyl-8-chloro-N10-(3, 4-xylyl)-5-deazaflavin VIIIb, N10-dodecyl-5deazaflavin VIIIc, 5-phenethyl-N10-n-octyl or n-dodecyl-5-deazaflavins VIIIf, g, pyridodipyrimidine derivative IXc, bis-(5-deazaflavin-10-yl)-nhexane Xa, and Shumisho database compound 831.

\section{Conclusion:}

3'and/or8'-disubstituted-5'-deaza-androsta[2, 3- g] pteridine-2', 4'(3' $\mathrm{H}$, 8'H)-diones VIa, b, and d, vertical bis-(5-deazaflavin-10-yl)-n-hexane Xa, exhibited remarkable non-selective PTK binding affinities. They presented the lowest $\Delta \mathrm{Gb}$ on docking against different classes of PTKs, i.e. -9.46 -13 . 28, and $-0.20--13.74 \mathrm{kcal} / \mathrm{mol}$. Hydrogen bonds were formed from ranging from one to four and zero to three with the distance of $1.60 \sim 4.49$ $\AA$, and $0.72 \sim 3.08$, respectively. Both of 2-substituted 2-deoxo-5deazaflavin and flavins VIIa, i. l, q, r and 5-unsubstituted and 5substituted-5-deazaflavins VIIIb, c, f, g also exhibited nonselective fine docking results within different PTKs. Furthermore, compounds IIa, IVc, $\mathrm{Vd}$, and 831 demonstrated to possess selective PTK binding affinities to certain classes of tyrosine kinases, namely: HGFR (c-met), ZAP-70, insulin receptor kinase, and EGFR, respectively. These findings revealed that hybrid compounds of 5-deazaflavins and steroids, that is, VIa, b, and VId would be promising candidates as antitumor agents.

Replacement of pyrimidoquinoline ring of 5-deazaflavins or benzopteridine ring of flavins with pyridodipyrimidines (IXc) as a new NAD-type redox catalyst produced compounds with excellent docking binding affinities wherein 2,7,8,10-tetraphenyl substitution enabled planar interaction within the target site so as to produce favorable docking affinity. Moreover, tyrosine containing tripeptide, Trp-Glu-Tyr-OH (Vd), was found to compose a platform to produce small lead compounds as PTK inhibitors which exhibit selective insulin receptor kinase binding affinity due to its close structural resemblance to the intact ligand of the insulin receptor kinase (pdb code: 1rqq).

The amino acid coupled with 2-deoxo-5-deazaflavin and flavins (VII l, q, r) has been shown to enhance remarkably the binding affinities in PTK. The higher affinity of these designed derivatives is presumably attributed to the formation of more hydrogen bonds (2-4 hydrogen bonds) between their 2-amino acid moieties and the binding site residues.

References:

[1] C Seidel et al. World J Urol Jan 22 (2011) [PMID: 21258806]

[2] J Schlessinger, Cell 103: 211 (2000) [PMID: 11057895]

[3] M Kontoyianni et al. J Med Chem. 47: 558 (2004) [PMID: 14736237]

[4] R Wang et al. J Med Chem. 46: 2287 (2003) [PMID: 12773034]

[5] C Bissantz et al. J Med Chem. 43: 4759 (2000) [PMID: 11123984]

[6] G Morris et al. J Comput Chem. 30: 2785 (2009) [PMID: 19399780]

[7] RA Lewis, J Med Chem. 48: 1638 (2005) [PMID: 15743205]

[8] TR Burke et al. Biopolymers 60: 32 (2001) [PMID: 11376431]

[9] MK Paul \& AK Mukhopadhyay, Int J Med Sci. 1: 101 (2004) [PMID: 15912202]

[10] S Noonberg \& CC Benz, Drugs, 59: 753 (2000)

[11] HI Ali et al. Bioorg Med Chem. 15: 242 (2007) [PMID: 17049252]

[12] T Nagamatsu et al. Nucleic Acid Symp Ser 52: 561 (2008) [PMID: 18776503]

[13] HI Ali et al. Bioorg Med Chem. 15: 242 (2007) [PMID: 17049252]

[14] HI Ali et al. Eur J Med Chem 43: 1376 (2008) [PMID: 18055068]

[15] HI Ali et al. Biorg Med Chem. 15: 6336 (2007) [PMID: 17644399]

[16] HI Ali et al. Biorg Med Chem. 16: 922 (2008) [PMID: 17962027]

[17] AR Shrestha et al. Biorg Med Chem. 16: 9161 (2008) [PMID: 18819815]

[18] Y Kanaoka et al. Bioorg Med Chem 6: 301 (1998) [PMID: 9568284]

[19] T Kimachi et al. Bioorg Med Chem 8: 2027 (2000) [PMID: 11003147]

[20] J Stamos et al. J Biol Chem. 277: 46265 (2002) [PMID: 12196540]

\footnotetext{
Edited by $P$ Kangueane Citation: Ali \& Akaho. Bioinformation 5(9): 368-374 (2011) License statement: This is an open-access article, which permits unrestricted use, distribution, and reproduction in any medium, for non-commercial purposes, provided the original author and source are credited.
} 


\section{Supplementary material:}

Table 1: AutoDock binding free energies $\left(\Delta \mathrm{G}_{\mathrm{b}}\right)$, inhibition constants (Ki), RMSD values, and hydrogen bonds of the top three ranks of the docked inhibitors against different protein tyrosine kinases

\begin{tabular}{|c|c|c|c|c|c|c|c|c|}
\hline \multirow[t]{2}{*}{ PDB code } & \multirow{2}{*}{\multicolumn{2}{|c|}{$\begin{array}{l}\text { Top ranked } \\
\text { inhibitors }\end{array}$}} & \multirow[t]{2}{*}{$\begin{array}{l}\Delta \mathrm{G}_{\mathrm{b}} \\
(\mathrm{kcal} / \mathrm{mol})\end{array}$} & \multirow[t]{2}{*}{$\mathrm{K}_{\mathrm{i}}$} & \multicolumn{3}{|c|}{$\begin{array}{l}\text { Hydrogen bonds between atoms of compounds and amino } \\
\text { acids of PTK }\end{array}$} & \multirow[t]{2}{*}{$\operatorname{RMSD}(\AA)$} \\
\hline & & & & & atom of comp. & amino acid & distance $(\AA)$ & \\
\hline \multirow{6}{*}{ 1agw } & 1) & VIIq & -11.85 & \multirow{3}{*}{ 2.05E-09 } & $\mathrm{COOH}$ & O of Asp641 & 1.05 & \multirow{3}{*}{5.44} \\
\hline & & & & & $2-\mathrm{NH}$ & O of Asp641 & 1.91 & \\
\hline & & & \multirow[b]{3}{*}{-11.51} & & $\mathrm{p}-\mathrm{OH}$ & O of Glu562 & 1.26 & \\
\hline & 2) & VIa & & \multirow{2}{*}{ 3.66E-09 } & 4-Oxо & HN of Lys514 & 1.79 & \multirow[b]{2}{*}{4.42} \\
\hline & & & & & $17-\mathrm{OH}$ & O of Glu571 & 2.33 & \\
\hline & 3) & VIb & -10.96 & $9.28 \mathrm{E}-09$ & 2-Oxо & HN of Lys514 & 2.05 & 4.49 \\
\hline \multirow{11}{*}{$1 \mathrm{~m} 17$} & 1) & IVc & & & $\mathrm{p}-\mathrm{OH}$ & O of Met769 & 1.94 & \multirow{5}{*}{3.54} \\
\hline & & & & & $\mathrm{m}-\mathrm{OH}$ & O of Gln 767 & 2.32 & \\
\hline & & & -10.77 & $1.28 \mathrm{E}-08$ & $\mathrm{~m}-\mathrm{O}$ & HN of Met769 & 2.18 & \\
\hline & & & & & piprazinyl-NH & O of Thr766 & 2.48 & \\
\hline & & & & & piprazinyl-NH & O of Ala719 & 2.15 & \\
\hline & 2) & VIb & -1066 & & $2-\mathrm{Oxo}$ & HN of Gly697 & 2.49 & 373 \\
\hline & & & -10.66 & $1.55 E-08$ & $17-\mathrm{OH}$ & O of Glu738 & 2.25 & 3.13 \\
\hline & 3) & VIIr & & & histadinyl-CO & HN of Asp831 & 1.91 & \\
\hline & & & -10.52 & 1 95F-08 & histadinyl-COO & HN of Lys721 & 2.20 & \\
\hline & & & -10.52 & $1.95 \mathrm{E}-08$ & imidazolyl-NH & O of Leu764 & 2.34 & 2.68 \\
\hline & & & & & imidazolyl-NH & O of Ala719 & 1.81 & \\
\hline & 1) & $\mathrm{Xa}$ & -11.90 & $1.90 \mathrm{E}-09$ & $4-\mathrm{Oxo}$ & HN of Met1160 & 2.15 & 0.72 \\
\hline & 2) & IIa & & & $\mathrm{p}-\mathrm{COOH}$ & O of Asp1231 & 2.19 & \\
\hline $1 \mathrm{r} 0 \mathrm{p}$ & & & -11.41 & 4.30E-09 & $\mathrm{m}-\mathrm{NH}$ & O of Asp1228 & 2.11 & 1.88 \\
\hline & & & & & $\mathrm{m}-\mathrm{NH}$ & O of Asp1228 & 2.07 & \\
\hline & 3) & 831 & -9.23 & 1.73E-07 & $\mathrm{p}-\mathrm{Cl}$ & HN of Lys 1110 & 2.49 & 0.87 \\
\hline & 1) & IVc & & & $\mathrm{p}-\mathrm{OH}$ & O of Thr1031 & 1.99 & \\
\hline & & & & & $\mathrm{m}-\mathrm{OH}$ & N of Tyr1031 & 2.33 & \\
\hline & & & -13.58 & $1.10 \mathrm{E}-10$ & $\mathrm{~m}-\mathrm{O}$ & HN of Tyr1031 & 2.43 & 4.86 \\
\hline & & & & & piprazinyl-NH & O of Asp1150 & 2.20 & \\
\hline & 2) & VId & -13.48 & $1.37 \mathrm{E}-10$ & $17-\mathrm{OH}$ & OH of Ser 1006 & 2.06 & 1.74 \\
\hline Irqq & 3) & Vd & & & $\mathrm{COOH}$ & O of Gln1004 & 2.28 & \\
\hline & & & & & $\mathrm{CO}$ & HN of Lys1030 & 1.85 & \\
\hline & & & -11.34 & 4.88E-09 & $\mathrm{COO}$ & HN of Lys1030 & 2.13 & 1.32 \\
\hline & & & & & indolyl-NH & O of Met1079 & 2.24 & \\
\hline & & & & & phenoxy p-O & HO of Ser1006 & 2.44 & \\
\hline & 1) & VIa & & & $2-\mathrm{Oxo}$ & HN of Arg62 & 2.13 & \\
\hline & & & & & N3-H & O of Ile71 & 2.29 & \\
\hline & & & -11.24 & $5.78 \mathrm{E}-09$ & $17-\mathrm{O}$ & HN of Arg32 & 2.19 & 2.95 \\
\hline & & & & & $17-\mathrm{O}$ & HN of Arg32 & 1.65 & \\
\hline $1 \mathrm{skj}$ & 2) & VIIa & & & 4-Oxо & HN of Lys60 & 1.87 & \\
\hline & & & & & 7-CO & HN of Lys60 & 2.06 & \\
\hline & & & -9.71 & $7.65 \mathrm{E}-08$ & 7-CO & HO of Thr36 & 2.33 & 2.35 \\
\hline & & & & & 7-CO & HO of Ser34 & 1.75 & \\
\hline & 3) & VIIIb & -8.09 & $1.18 \mathrm{E}-06$ & & ---a & & 1.79 \\
\hline & 1) & VIb & & & 2-Охо & HN of Ala 451 & 2.14 & \\
\hline & & & -11.39 & 4.47E-09 & 17-OH & N of Gly380 & 2.24 & 2.24 \\
\hline & & & & & $17-\mathrm{OH}$ & O of Gly383 & 1.88 & \\
\hline $1 x b b$ & 2) & VId & -10.79 & $1.24 \mathrm{E}-08$ & $17-\mathrm{OH}$ & O of Gly383 & 1.67 & 1.60 \\
\hline & 3) & $\mathrm{Xa}$ & & & 4-OxoTricycleA & HN of Phe382 & 2.31 & \\
\hline & & & -10.61 & 1.67E-08 & 4-OxoTricycleA & HN of Asn381 & 2.08 & 2.63 \\
\hline & & & & & 4-OxoTricycleB & HN of Ala451 & 1.79 & \\
\hline & 1) & VIIi & -15.12 & 8.31E-12 & & $---a$ & & 4.22 \\
\hline & 2) & $\mathrm{Xa}$ & -12.62 & $5.61 \mathrm{E}-10$ & & ---a & & 1.42 \\
\hline It46 & 3) & VIIl & -1132 & & TyrosinylCOOH & OH of Thr670 & 2.26 & \\
\hline & & & -11.32 & $5.07 \mathrm{E}-09$ & TyrosinylCOOH & N of Thr670 & 2.31 & 4.29 \\
\hline & 1) & $\mathrm{Xa}$ & & & 4-OxoTricycleA & HN of Lys866 & 2.10 & \\
\hline & & & -10.20 & $3.33 E-08$ & 4-OxoTricycleB & HN of Asn921 & 1.98 & 1.06 \\
\hline 1y6a & 2) & VIIIf & -9.75 & 7.18E-08 & 4-Oxо & HN of Asn921 & 2.44 & 102 \\
\hline губа & & & -9.15 & $1.18 \mathrm{E}-08$ & 4-Oxо & HN of Asn921 & 2.36 & 1.02 \\
\hline & 3) & VId & $-9,46$ & & $17-\mathrm{OH}$ & O of Glu915 & 1.78 & 1.78 \\
\hline & & & -9.46 & $1.16 \mathrm{E}-0 /$ & $17-\mathrm{OH}$ & HN of Asn921 & 1.79 & $1 . / 8$ \\
\hline $1 \mathrm{fpu}$ & 1) & $\mathrm{Xa}$ & -13.74 & $8.45 \mathrm{E}-11$ & & $---a$ & & 3.08 \\
\hline
\end{tabular}




\section{Bioinformation Volume 5}

open access

www.bioinformation.net

Issue 9

Hypothesis

\begin{tabular}{|c|c|c|c|c|c|c|c|c|}
\hline \multirow{5}{*}{$1 q c f$} & 2) & VIb & -11.44 & 4.13E-09 & $17-\mathrm{OH}$ & O of Tyr253 & 2.34 & 3.94 \\
\hline & 3) & VIIIb & -11.09 & $7.40 \mathrm{E}-09$ & 2-Oxо & HO of Thr315 & 1.77 & 4.49 \\
\hline & 1) & VIIIg & -11.52 & 3.62E-09 & 2-Oxо & HN of Lys295 & 1.59 & 1.06 \\
\hline & 2) & VIIIc & -11.31 & 5.11E-09 & 4-Oxо & HN of Met 341 & 1.72 & 3.99 \\
\hline & 3) & VIb & -11.09 & 7.44E-09 & $\begin{array}{l}17-\mathrm{OH} \\
17-\mathrm{O}\end{array}$ & $\begin{array}{l}\text { O of Glu339 } \\
\text { HN of Met341 }\end{array}$ & $\begin{array}{l}2.08 \\
2.05\end{array}$ & 3.02 \\
\hline \multirow{3}{*}{$1 \mathrm{u} 59$} & 1) & VIa & -11.10 & 7.33E-09 & N3-H & O of Glu386 & 2.26 & 2.48 \\
\hline & 2) & IIb & -10.52 & 1.93E-08 & $\begin{array}{l}\mathrm{p}-\mathrm{CO} \\
\mathrm{m}-\mathrm{NO}\end{array}$ & $\begin{array}{l}\text { HN of His } 423 \\
\text { HN of Lys } 424\end{array}$ & $\begin{array}{l}2.17 \\
1.70\end{array}$ & 1.99 \\
\hline & 3) & VIIr & -10.06 & 4.24E-08 & $\begin{array}{l}4-\mathrm{Oxo} \\
5-\mathrm{N}-\mathrm{O}\end{array}$ & $\begin{array}{l}\text { HN of Ala417 } \\
\text { HN of Ala417 }\end{array}$ & $\begin{array}{l}1.93 \\
2.90\end{array}$ & 1.63 \\
\hline \multirow{3}{*}{$1 \mathrm{yvj}$} & 1) & VId & -12.48 & 7.13E-10 & $17-\mathrm{OH}$ & O of Gly831 & 2.35 & 2.10 \\
\hline & 2) & $\mathrm{Xa}$ & -12.36 & 8.76E-10 & 4-OxoTricycleA & HN of Lys855 & 2.31 & 1.03 \\
\hline & 3) & VIIIf & -10.81 & $1.18 \mathrm{E}-08$ & 4-Охо & HN of Lys855 & 2.33 & 3.18 \\
\hline
\end{tabular}

1) to 3 ) represent top ranked three compounds based on their binding free energies. a represent that no hydrogen bond was detected. 